\title{
THE COUPLED/QUASI-STATIC APPROXIMATION IN ONE-DIMENSIONAL LINEAR THERMOELASTICITY
}

\author{
BY \\ RICHARD J. WEINACHT \\ Mathematical Sciences Department, University of Delaware, Newark, DE
}

1. Introduction. Quasi-static approximations in continuum mechanics are very useful and widely used; in particular, this is true in thermoelasticity (see, e.g., [1], much of which is devoted to quasi-static considerations). In this paper we consider the coupled/ quasi-static (C/QS) approximation in linear thermoelasticity for a homogeneous onedimensional continuum.

Following Day [3] we use the term "coupled/quasi-static approximation" to mean that the inertial term in the momentum equation (Eq. (1.2) below) is neglected, but the terms of thermomechanical coupling are retained in both the momentum equation and the energy equation (Eq. (1.1) below). Thus in (1.2) one puts the inertial constant $\varepsilon^{2}$ equal to zero but retains a nonzero coupling constant $\gamma$ in (1.1) and (1.2). Since a second time-derivative term is thereby neglected it is not surprising that the quasi-static approximations of the displacement and temperature are not uniformly asymptotically correct in space and time (without "corrector terms" as in $[7,4]$ for the equation of Cattaneo and nonlinear generalizations) to lowest order in the inertial constant. A principal result of this paper is that, perhaps surprisingly, the quasi-static approximation of the entropy is uniformly asymptotically correct in space and time (on $[0,1] \times[0, T]$ for any given positive $T$ ) to lowest order, consisting solely of diffusion-type terms.

As emphasized in [5], Day has shown that $\hat{\theta}$, the C/QS approximation of the temperature $\theta$, satisfies

$$
|\theta-\hat{\theta}| \rightarrow 0 \quad \text { as } t \rightarrow \infty
$$

uniformly in space (on $[0,1])$. The same is true if $\hat{\theta}$ is replaced by $\tilde{\theta}$, the temperature given by the classical heat equation that corresponds to the uncoupled/quasi-static approximation. We are not asserting that quasi-static approximations are not useful but merely that they are not usually uniformly asymptotically correct in space and time. The uniform result for entropy considered here and in [5] is exceptional.

\footnotetext{
Received June 8, 1998.

2000 Mathematics Subject Classification. Primary 35B25, 74A15, 74F05, 35K05, 74B05, 35Q72.

This work was supported in part by GNAFA-CNR of the Consiglio Nazionale delle Ricerche of Italy.

E-mail address: weinacht@math.udel.edu
} 
The governing system of PDEs (in non-dimensional form) of one-dimensional linear thermoelasticity is

$$
\begin{aligned}
& \Pi(\theta, u):=\theta_{t}-\theta_{x x}+\gamma u_{x t}=0, \\
& H_{\varepsilon}(\theta, u):=\varepsilon^{2} u_{t t}-u_{x x}+\gamma \theta_{x}=0,0<x<1,
\end{aligned}
$$

where $\theta$ is the dimensionless temperature and $u$ is the dimensionless displacement of an elastic bar (or equivalently an infinite slab of thickness 1). The two positive parameters $\varepsilon^{2}$ and $\gamma$ are, respectively, the inertial constant and the coupling constant. We consider the initial/boundary value problem (IBVP) for (1.1)-(1.2) with initial conditions

$$
\begin{aligned}
\theta(x, 0 ; \varepsilon) & =\phi(x), \\
u(x, 0 ; \varepsilon) & =f(x), \\
u_{t}(x, 0 ; \varepsilon) & =g(x)
\end{aligned}
$$

which give the initial temperature, displacement, and velocity (independent of $\varepsilon$ ) along with the Dirichlet boundary conditions

$$
\begin{aligned}
& \theta(0, t ; \varepsilon)=\theta(1, t ; \varepsilon)=0, \\
& u(0, t ; \varepsilon)=u(1, t ; \varepsilon)=0,
\end{aligned}
$$

which state that the ends of the bar are fixed and the temperature is maintained at zero at the ends of the bar.

The coupled/quasi-static approximation of (1.1)-(1.7) consists of setting $\varepsilon=0$ in (1.2) to arrive at the system

$$
\begin{aligned}
\Pi(\hat{\theta}, \hat{u}) & :=\hat{\theta}_{t}-\hat{\theta}_{x x}+\gamma \hat{u}_{x t}=0, \\
H_{0}(\hat{\theta}, \hat{u}) & :=-\hat{u}_{x x}+\gamma \hat{\theta}_{x}=0
\end{aligned}
$$

plus the same boundary conditions as (1.6)-(1.7)

$$
\begin{aligned}
& \hat{\theta}(0, t)=\hat{\theta}(1, t)=0, \\
& \hat{u}(0, t)=\hat{u}(1, t)=0
\end{aligned}
$$

plus precisely one of the initial conditions (1.3)--(1.4). The resulting IBVP has at most one (smooth) solution as can be seen via Energy Estimates (see below in Sec. 4). If one is primarily interested in the evolution of the temperature, then one would choose the initial condition

$$
\hat{\theta}(x, 0)=\phi(x),
$$

but it should be emphasized that it is possible to consider different C/QS approximations depending on the choice of initial condition as we do below.

Various aspects of the C/QS approximation (as well as the uncoupled case) have been treated in a series of papers of W. A. Day (see his monograph [3] and references therein). Our scaling for dimensionless quantities is the same as used by Day [3] but our notation simplifies some of our resulting formulas (our $\gamma$ is Day's $\sqrt{a}$ and our $\varepsilon^{2}$ is Day's $b$ ). Esham and Weinacht [5] obtained results similar to those presented here in the case of boundary 
conditions which greatly simplify the analysis. A key element of our work is the repeated use of energy estimates, which was the subject of [11].

For recent results on various aspects of thermoelasticity since the pioneering work of Dafermos [2] in the linear case and Slemrod [10] in the nonlinear case, we refer the reader to the references in Rivera [9] for the linear case and Racke/Shibata [8] for the nonlinear case. We note that [8] treats the more difficult case of Dirichlet boundary conditions as we do here.

2. The C/QS approximation. The solution of the IBVP (1.8)-(1.12) can be explicitly given in several ways (cf. [3]). Here we do so in a form that is suitable for generalization to higher dimensions. Firstly, from (1.9) and (1.11) we have

$$
\hat{u}(x, t)=-\gamma\left(\Gamma \hat{\theta}_{x}\right)(x, t)
$$

where $\Gamma$ is the one-dimensional Green's operator that inverts the boundary value problem

$$
\left\{\begin{array}{l}
-y^{\prime \prime}=\psi(x), \quad 0<x<1 \\
y(0)=y(1)=0
\end{array}\right.
$$

so that

$$
y(x)=(\Gamma \psi)(x)
$$

for arbitrary continuous $\psi$. The operator $\Gamma$ is a bounded linear operator from the Sobolev space $H^{-1}(0,1)$ to $H_{0}^{1}(0,1)$ whose restriction to $L_{2}(0,1)$ is compact.

We now make some preparations to obtain an equation for $\hat{\theta}$ alone. Note from $(2.1)$ that

$$
\hat{u}_{x}=-\gamma\left(\partial_{x} \Gamma \partial_{x}\right) \hat{\theta}=-\gamma B \hat{\theta}
$$

where $B:=\partial_{x} \Gamma \partial_{x}$ is a bounded linear non-local operator from $L_{2}(0,1)$ to $L_{2}(0,1)$. Moreover, $B$ is selfadjoint and negative semi-definite. To establish these latter assertions let us here and below write

$$
(u, v):=\int_{0}^{1} u(x) v(x) d x
$$

for the $L_{2}$-inner product and $\langle\cdot, \cdot\rangle$ for the duality pairing for $H^{-1}(0,1)$ and $H_{0}^{1}(0,0)$. Thus for any $u$ in $L_{2}(0,1)$ and $\psi$ in $H_{0}^{1}(0,1)$,

$$
\left\langle\partial_{x} u, \psi\right\rangle=-\left(u, \partial_{x} \psi\right)
$$

Then we see that $B$ is selfadjoint since

$$
\begin{aligned}
(B u, v) & =\left(\partial_{x} \Gamma \partial_{x} u, v\right)=-\left\langle\partial_{x} v, \Gamma \partial_{x} u\right\rangle \\
& =-\left\langle\partial_{x} u, \Gamma \partial_{x} v\right\rangle=\left(u, \partial_{x} \Gamma \partial_{x} v\right)=(u, B v) .
\end{aligned}
$$

Similarly $B$ is negative semi-definite because

$$
(B u, u)=-\left\langle\partial_{x} u, \Gamma \partial_{x} u\right\rangle \leq 0
$$

since $\Gamma$ is a positive-definite operator $(B$ is not negative definite since any constant function is mapped by $B$ into zero; in fact, $(B u, u)=0$ only for $u \equiv$ constant a.e.). 
Substitution of (2.1) into (1.8) yields

$$
0=\hat{\theta}_{t}-\hat{\theta}_{x x}-\gamma^{2} B \hat{\theta}_{t},
$$

or

$$
A \hat{\theta}_{t}-\hat{\theta}_{x x}=0
$$

where

$$
A=I-\gamma^{2} B \text {. }
$$

Thus $A$ is a non-local bounded selfadjoint linear operator from $L_{2}(0,1)$ to $L_{2}(0,1)$. It is also positive-bounded-below since

$$
(A u, u)=(u, u)-\gamma^{2}(B u, u) \geq(u, u) .
$$

To obtain an explicit representation for a solution $\{\hat{\theta}, \hat{u}\}$ of $(1.8)-(1.12)$ we separate variables in $(2.2)$

$$
\hat{\theta}(x, t)=\widehat{\Theta}(t) \omega(x)
$$

so that we are led to the eigenvalue problem

$$
\left\{\begin{array}{l}
-\omega^{\prime \prime}=\lambda A \omega, \quad 0<x<1, \\
\omega(0)=\omega(1)=0 .
\end{array}\right.
$$

By application of $\Gamma$ we see that this eigenvalue problem is equivalent to that for the operator equation

$$
\omega=\lambda \Gamma A \omega
$$

for the compact operator $\Gamma A$ on $L_{2}(0,1)$. Although $\Gamma$ and $A$ are selfadjoint, the product $\Gamma A$ is not and so we introduce a new inner product on $L_{2}(0,1)$ weighted by $A$,

$$
(u, v)_{A}:=\int_{0}^{1} u(x) A v(x) d x,
$$

and call the resulting space $L_{A}^{2}(0,1)$. On this space we have an eigenvalue problem for the compact operator $K:=\Gamma A$ which is selfadjoint since

$$
(K u, v)_{A}=(\Gamma A u, A v)=(u, K v)_{A} .
$$

Moreover, $K$ is positive definite on $L_{A}^{2}(0,1)$ because

$$
(K u, u)_{A}=(\Gamma A u, A u) .
$$

Thus (the nondegenerate) $K$ has an infinite sequence of positive (reciprocal) eigenvalues

$$
0<\lambda_{1} \leq \lambda_{2} \leq \cdots \leq \lambda_{n}<\cdots \rightarrow \infty
$$

with corresponding $C^{\infty}$-smooth eigenfunctions $\omega_{1}, \omega_{2}, \ldots$ that are orthonormal,

$$
\left(\omega_{l}, \omega_{m}\right)_{A}=\delta_{l m},
$$

and complete in $L_{A}^{2}(0,1)$. 
There are several useful relations that follow from the orthonormality of the $\left\{\omega_{l}\right\}$ in $L_{A}^{2}(0,1)$ :

$$
\begin{gathered}
\left(\omega_{l}^{\prime}, \omega_{m}^{\prime}\right)=-\left(\omega_{l}^{\prime \prime}, \omega_{m}\right)=\lambda_{l}\left(A \omega_{l}, \omega_{m}\right)=\lambda_{l} \delta_{l m}, \\
\gamma\left(v_{l}, \omega_{m}^{\prime}\right)=\gamma^{2}\left(B \omega_{l}, \omega_{m}\right)=\left((I-A) \omega_{l}, \omega_{m}\right)=\left(\omega_{l}, \omega_{m}\right)-\delta_{l m}
\end{gathered}
$$

so that

$$
\left(v_{l}^{\prime \prime}, v_{m}\right)=\gamma\left(\omega_{l}^{\prime}, v_{m}\right)=\delta_{l m}-\left(\omega_{l}, \omega_{m}\right) .
$$

It will be useful below to introduce the $N$-by- $N$ symmetric Gram matrices $\Omega$ and $V$ defined via

$$
\Omega_{m l}=\left(\omega_{m}, \omega_{l}\right), \quad V_{m l}=\left(v_{m}, v_{l}\right)
$$

which are positive definite. Since $(A-I)=-\gamma^{2} B$ is positive semi-definite on $L_{2}(0,1)$, so is its projection on the span of $\left\{\omega_{1}, \ldots, \omega_{N}\right\}$ which corresponds to the matrix $(I-\Omega)$. More precisely,

$$
\omega=\sum_{l=1}^{N} \alpha_{l} \omega_{l} \Rightarrow 0 \leq((A-I) \omega, \omega)=\boldsymbol{\alpha}^{\mathrm{T}}(I-\Omega) \boldsymbol{\alpha}
$$

where $\boldsymbol{\alpha}=\left(\alpha_{1}, \ldots, \alpha_{N}\right)^{\mathrm{T}}$ and equality holds if and only if $\omega$ is constant (a.e.). But on this finite-dimensional space, $\omega$ is constant only for $\omega=0$. Thus $(I-\Omega)$ is positive definite.

We are now ready to complete the solution of (1.8)-(1.12). Expand $\hat{\theta}$ :

$$
\hat{\theta}(x, t):=\sum_{l=1}^{\infty} \widehat{\Theta}_{l}(t) w_{l}(x)
$$

with

$$
\widehat{\Theta}_{l}(t)=\alpha_{l} e^{-\lambda_{l} t}
$$

and the Fourier coefficient $\alpha_{l}$ given by

$$
\alpha_{l}=\left(\phi, w_{l}\right)_{A}
$$

where convergence in $(2.6)$ is in $L_{A}^{2}(0,1)$ for each fixed $t$ in $(0, \infty)$.

Then $\hat{u}$ is uniquely defined by $(2.1)$, i.e.,

$$
\hat{u}(x, t)=-\gamma\left(\Gamma \hat{\theta}_{x}\right)(x, t)
$$

in terms of the $\hat{\theta}$ constructed above. It is interesting that the C/QS approximation of the entropy

$$
\hat{\eta}:=\hat{\theta}+\gamma \hat{u}_{x}=\hat{\theta}-\gamma^{2} B \hat{\theta}=A \hat{\theta}
$$

is expressed directly in terms of $\hat{\theta}$ through the non-local operator $A$. Thus, if one is primarily interested in the entropy, then the choice of the initial condition (in place of (1.12))

$$
\hat{\theta}(x, 0)=A^{-1}\left[\phi+\gamma f^{\prime}\right](x)
$$

is more appropriate since we then have

$$
\hat{\eta}(x, 0)=\phi(x)+\gamma f^{\prime}(x),
$$


which is the correct initial condition for the entropy in the full thermoelastic problem (1.1)-(1.7). If the above choice is made, then

$$
\begin{aligned}
\hat{\eta}(x, t)=A \hat{\theta}(x, t) & =\sum_{l=1}^{\infty}\left(A^{-1}\left[\phi+\gamma f^{\prime}\right], A w_{l}\right) A w_{l} \\
& =\sum_{l=1}^{\infty}\left(\phi+\gamma f^{\prime}, w_{l}\right) A w_{l} .
\end{aligned}
$$

Following this thought concerning conditions in the full thermoelastic problem, consider the $N$-th order Faedo/Galerkin approximation of $\hat{\theta}$ and $\hat{u}$,

$$
\begin{aligned}
& \hat{\theta}^{N}(x, t):=\sum_{l=1}^{N} \widehat{\Theta}_{l}^{N}(t) w_{l}(x), \\
& \hat{u}^{N}(x, t):=\sum_{l=1}^{N} \widehat{U}_{l}^{N}(t) v_{l}(x)
\end{aligned}
$$

with the stipulation that, for $m=1, \ldots, N$,

$$
\begin{gathered}
\left(\Pi\left(\hat{\theta}^{N}, \hat{u}^{N}\right), w_{m}\right)=0 \\
\left(H_{0}\left(\hat{\theta}^{N}, \hat{u}^{N}\right), v_{m}\right)=0
\end{gathered}
$$

where we emphasize that the inner product is in $L_{2}(0,1)$ not $L_{A}^{2}(0,1)$. By use of $(2.3)-$ (2.5) the orthogonality conditions $(2.10)-(2.11)$ can be written

$$
\begin{gathered}
\Omega \dot{\hat{\boldsymbol{\Theta}}}+\Lambda \widehat{\boldsymbol{\Theta}}+(I-\Omega) \dot{\hat{\mathbf{U}}}=\mathbf{0} \\
-(I-\Omega)[\widehat{\mathbf{U}}-\widehat{\boldsymbol{\Theta}}]=\mathbf{0}
\end{gathered}
$$

where $\widehat{\Theta}$ (resp. $\widehat{\mathrm{U}})$ is the $N$-vector with components $\widehat{\Theta}_{l}^{N}$ (resp. $\widehat{U}_{l}^{N}$ ). From $(2.12)-(2.13)$ one finds that

$$
\dot{\widehat{\Theta}}+\Lambda \widehat{\Theta}=0
$$

which is a diagonal system so that

$$
\widehat{\Theta}_{l}^{N}=\alpha_{l} e^{-\lambda l t}
$$

in consonance with the result from separation of variables in (2.7). We will compare this result later with that for the Galerkin approximation for the full thermoelastic problem.

We add here some completeness results including the

LEmma 2.1. The following sequences of functions are complete (三total) in the spaces indicated:

(a) $\left\{\omega_{l}\right\}$ and $\left\{v_{l}\right\}$ in $L_{2}(0,1)$,

(b) $\left\{\omega_{l}\right\}$ and $\left\{v_{l}\right\}$ in $H_{0}^{1}(0,1)$.

Proof. Of course, the assertion (a) follows from (b) since the norm of $H_{0}^{1}$ is stronger than that of $L_{2}$, but it is convenient to prove (a) first and use that result in the proof of (b). For the proof of (a), if $\psi$ is in $L_{2}(0,1)$ and orthogonal to all the $\omega_{l}$, then

$$
0=\left(\psi, \omega_{l}\right)=\left(\psi, A^{-1} A \omega_{l}\right)=\left(A^{-1} \psi, A \omega_{l}\right)
$$


which implies that $A^{-1} \psi=0$ and hence $\psi=0$. Similarly for any $w$ in $L_{2}(0,1)$ we have

$$
0=\left(w, v_{l}\right)=-\gamma\left(w, \Gamma \omega_{l}^{\prime}\right)=-\gamma\left(\Gamma w, \omega_{l}^{\prime}\right)=\gamma\left(\partial_{x} \Gamma w, \omega_{l}\right)
$$

for $l=1,2, \ldots$ Then $\Gamma w=$ constant and the constant must be zero since $\Gamma w$ vanishes at $x=0$ and 1. But $\Gamma w=0 \Rightarrow w=0$ and the $\left\{v_{l}\right\}$ are complete in $L_{2}(0,1)$.

For (b) let $\psi$ be in $H_{0}^{1}(0,1)$ such that for $l=1,2, \ldots$,

$$
\begin{aligned}
0 & =\left(\psi, \omega_{l}\right)+\left(\psi^{\prime}, \omega_{l}^{\prime}\right)=\left(A^{-1} \psi, A \omega_{l}\right)-\left(\psi, \omega_{l}^{\prime \prime}\right) \\
& =\left(A^{-1} \psi, A \omega_{l}\right)+\lambda_{l}\left(\psi, A \omega_{l}\right)=\left(A^{-1} \psi+\lambda_{l} \psi, A \omega_{l}\right) \\
& =\left(A^{-1} \psi+\lambda_{l} \psi, \omega_{l}\right)_{A}
\end{aligned}
$$

from which it follows that $\psi+\lambda_{l} A \psi=0$ and hence

$$
(\psi, \psi)+\lambda_{l}(\psi, A \psi)=0,
$$

which implies that $\psi=0$. For $w$ in $H_{0}^{1}(0,1)$ such that for all $l=1,2, \ldots$

$$
\begin{aligned}
0 & =\left(w, v_{l}\right)+\left(w^{\prime}, v_{l}^{\prime}\right)=-\gamma\left(w, \Gamma \omega_{l}^{\prime}\right)-\left(w, v_{l}^{\prime \prime}\right) \\
& =-\gamma\left(\Gamma w+w, \omega_{l}^{\prime}\right)
\end{aligned}
$$

and hence $\Gamma w+w=0$ (since its trace is zero at $x=0$ and 1 ). Thus $(\Gamma w+w, w)=0$ and $w=0$. With this the proof of the lemma is complete.

Finally, we note that the eigenvalues $\left\{\lambda_{l}\right\}$ and the eigenfunctions $\left\{\omega_{l}, v_{l}\right\}$ are explicitly known up to the solution of a transcendental equation and have been given previously by Day [3]. For convenience of the reader we list them here. For $l=1,2, \ldots$,

$$
\begin{gathered}
\lambda_{2 l}=\left(\frac{2 l \pi}{c}\right)^{2}, \quad \omega_{2 l}(x)=\frac{\sqrt{2}}{c} \sin (2 l \pi x), \\
\omega_{2 l-1}(x)=\alpha_{2 l-1}\left\{\sin \left(\mu_{2 l-1}\right)\left[\cos \left(\mu_{2 l-1} x\right)-1\right]+\left(1-\cos \mu_{2 l-1}\right) \sin \left(\mu_{2 l-1} x\right)\right\}
\end{gathered}
$$

where $\alpha_{2 l-1}$ are constants that make $\left(\omega_{2 l-1}, \omega_{2 l-1}\right)_{A}=1$ and the $\mu_{2 l-1}:=c \sqrt{\lambda_{2 l-1}}$ are solutions of the transcendental equation

$$
2 \gamma^{2} \tan \left(\frac{\mu_{2 l-1}}{2}\right)+\mu_{2 l-1}=0 .
$$

The corresponding $v_{l}$ are obtained from $\omega_{l}$ via (2.1) so that

$$
v_{2 l}(x)=\frac{\sqrt{2}}{c} \frac{\gamma}{2 l \pi}[1-\cos (2 l \pi x)]
$$

and

$$
\begin{aligned}
v_{2 l-1}(x)=\alpha_{2 l-1} \frac{\gamma}{\mu_{2 l-1}}\{ & \left(1-\cos \mu_{2 l-1}\right)\left[1-\cos \left(\mu_{2 l-1} x\right)\right] \\
& \left.+\frac{1}{\gamma^{2}} \sin \left(\mu_{2 l-1}\right)\left[\gamma^{2} \sin \left(\mu_{2 l-1} x\right)+\mu_{2 l-1} x\right]\right\} .
\end{aligned}
$$


3. The full thermoelastic problem. Here we consider the IBVP (with $\varepsilon>0$ ) (1.1)-(1.7) which we attack by the method of Faedo/Galerkin. Our Ansatz for the $N$-th order Galerkin approximation is

$$
\begin{aligned}
& \theta^{N}(x, t ; \varepsilon)=\sum_{l=1}^{N} \Theta_{l}^{N}(t ; \varepsilon) \omega_{l}(x), \\
& u^{N}(x, t ; \varepsilon)=\sum_{l=1}^{N} U_{l}^{N}(t ; \varepsilon) v_{l}(x)
\end{aligned}
$$

where the $\omega_{l}, v_{l}$ are the eigenfunctions of the C/QS approximation of the previous section. Essentially, we want $\Pi\left(\theta^{N}, u^{N}\right)$ to be orthogonal to $\left\{\omega_{1}, \ldots, \omega_{N}\right\}$ in the sense of $L_{2}(0,1)$ (not $\left.L_{A}^{2}(0,1)\right)$ and $H_{\varepsilon}\left(\theta^{N}, u^{N}\right)$ to be orthogonal to $\left\{v_{1}, \ldots, v_{N}\right\}$ in $L_{2}(0,1)$. Thus we require that for $m=1, \ldots, N$,

$$
\begin{aligned}
& \sum_{l=1}^{N}\left\{\dot{\Theta}_{l}^{N}\left(\omega_{l}, \omega_{m}\right)+\Theta_{l}^{N}\left(\omega_{l}^{\prime}, \omega_{m}^{\prime}\right)-\gamma \dot{U}_{l}^{N}\left(v_{l}, \omega_{m}^{\prime}\right)\right\}=0 \\
& \sum_{l=1}^{N}\left\{\varepsilon^{2} \ddot{U}_{l}^{N}\left(v_{l}, v_{m}\right)+U_{l}^{N}\left(v_{l}^{\prime}, v_{m}^{\prime}\right)+\gamma \Theta_{l}^{N}\left(\omega_{l}^{\prime}, v_{m}\right)\right\}=0
\end{aligned}
$$

which is a coupled system of $2 N$ ODEs.

To write this system compactly in vector form we use the $N$-by- $N$ (constant symmetric and positive definite) Gram matrices $\Omega$ and $V$ introduced in Sec. 2 and the diagonal matrix $\Lambda=\operatorname{diag}\left(\lambda_{1}, \ldots, \lambda_{N}\right)$. Then for vectors $\Theta^{N}=\left(\Theta_{1}^{N}, \ldots, \Theta_{N}^{N}\right)^{\mathrm{T}}$ and $\mathbf{U}=\left(U_{1}^{N}, \ldots, U_{N}^{N}\right)^{\mathrm{T}}$ the system $(3.1)-(3.2)$ can be written

$$
\begin{gathered}
\widetilde{\Pi}\left[\boldsymbol{\Theta}^{N}, \mathbf{U}^{N}\right]:=\Omega \dot{\boldsymbol{\Theta}}^{N}+\Lambda \boldsymbol{\Theta}^{N}+(I-\Omega) \dot{\mathbf{U}}^{N}=\mathbf{0}, \\
\widetilde{H}_{\varepsilon}\left[\boldsymbol{\Theta}^{N}, \mathbf{U}^{N}\right]:=\varepsilon^{2} V \ddot{\mathbf{U}}^{N}+(I-\Omega)\left[\mathbf{U}^{N}-\boldsymbol{\Theta}^{N}\right]=\mathbf{0}
\end{gathered}
$$

by use of (2.3)-(2.5). When $\varepsilon=0$ this system, of course, is (2.12)-(2.13).

We can obtain an initial-value problem for (3.3)-(3.4) by adjoining the initial conditions (independent of $\varepsilon$ )

$$
\Theta^{N}(0 ; \varepsilon)=\phi^{N}, \quad \mathbf{U}^{N}(0 ; \varepsilon)=\mathbf{f}^{N}, \quad \dot{\mathbf{U}}^{N}(0 ; \varepsilon)=\mathbf{g}^{N}
$$

where for $l=1, \ldots, N$ the components $\phi_{l}^{N}, f_{l}^{N}, g_{l}^{N}$ are obtained from the projections onto the subspaces of $L_{2}(0,1)$ spanned by $\left\{\omega_{1}, \ldots, \omega_{n}\right\}$ and $\left\{v_{1}, \ldots, v_{n}\right\}$. Thus, in an obvious notation,

$$
\begin{gathered}
\Pi^{N} \phi=\sum_{l=1}^{N} \phi_{l}^{N} \omega_{l}, \\
P^{N} f=\sum_{l=1}^{N} f_{l}^{N} v_{l}, \quad P^{N} g=\sum_{l=1}^{N} g_{l}^{N} v_{l} .
\end{gathered}
$$

The IVP (3.3)-(3.5) has a unique global solution $\left\{\Theta^{N}, \mathbf{U}^{N}\right\}$ which is $C^{\infty}$-smooth and we wish to examine convergence of $\left\{\theta^{N}, u^{N}\right\}$ as $N$ tends to infinity for fixed positive $\varepsilon$. This will be done in the Appendix with the aid of energy estimates provided in the next section. 
4. Some energy identities. Let $\left\{\boldsymbol{\Theta}^{N}, \mathbf{U}^{N}\right\}$ be solutions of the IVP (3.3)-(3.5). Then

$$
\begin{aligned}
& \sum_{l=1}^{N}\left\{\dot{\Theta}_{l}^{N}\left(\omega_{l}, \omega_{m}\right)+\Theta_{l}^{N}\left(\omega_{l}^{\prime}, \omega_{m}^{\prime}\right)-\gamma \dot{U}_{l}^{N}\left(v_{l}, \omega_{m}^{\prime}\right)\right\}=0 \\
& \sum_{l=1}^{N}\left\{\varepsilon^{2} \ddot{U}_{l}^{N}\left(v_{l}, v_{m}\right)+U_{l}^{N}\left(v_{l}^{\prime}, v_{m}^{\prime}\right)+\gamma \Theta_{l}^{N}\left(\omega_{l}^{\prime}, v_{m}\right)\right\}=0 .
\end{aligned}
$$

Multiplying (4.1) by $\Theta_{m}^{N}$ (respectively (4.2) by $\dot{U}_{m}^{N}$ ) and summing on $m$ from 1 to $N$ yields

$$
\begin{gathered}
\left(\partial_{t} \theta^{N}, \theta^{N}\right)+\left(\partial_{x} \theta^{N}, \partial_{x} \theta^{N}\right)-\gamma\left(\partial_{t} u^{N}, \partial_{x} \theta^{N}\right)=0, \\
\varepsilon^{2}\left(\partial_{t}^{2} u^{N}, \partial_{t} u^{N}\right)+\left(\partial_{x} u^{N}, \partial_{t} \partial_{x} u^{N}\right)+\gamma\left(\partial_{x} \theta^{N}, \partial_{t} u^{N}\right)=0,
\end{gathered}
$$

and so, by adding and integrating with respect to $t$ from 0 to $T$,

$$
E(T)+\int_{0}^{T}\left(\partial_{x} \theta^{N}, \partial_{x} \theta^{N}\right) d t=E(0)
$$

where $E$ is the energy defined by

$$
E(t)=\frac{1}{2}\left\{\left(\theta^{N}, \theta^{N}\right)+\varepsilon^{2}\left(\partial_{t} u^{N}, \partial_{t} u^{N}\right)+\left(\partial_{x} u^{N}, \partial_{x} u^{N}\right)\right\}
$$

Equation (4.3) is the first of our energy identities. An energy identity of higher order (in time) can be obtained in a similar way as follows. Differentiate (4.1) and (4.2) with respect to $t$. Then multiply the first of these by $\dot{\Theta}_{m}^{N}$ and the second by $\ddot{U}_{m}^{N}$ and sum on $m$ from 1 to $N$ to get

$$
\begin{gathered}
\left(\partial_{t}^{2} \theta^{N}, \partial_{t} \theta^{N}\right)+\left(\partial_{t} \partial_{x} \theta^{N}, \partial_{t} \partial_{x} \theta^{N}\right)-\gamma\left(\partial_{t}^{2} u^{N}, \partial_{t} \partial_{x} \theta^{N}\right)=0, \\
\varepsilon^{2}\left(\partial_{t}^{3} u^{N}, \partial_{t}^{2} u^{N}\right)+\left(\partial_{t} \partial_{x} u^{N}, \partial_{t}^{2} \partial_{x} u^{N}\right)+\gamma\left(\partial_{t} \partial_{x} \theta^{N}, \partial_{t}^{2} u^{N}\right)=0,
\end{gathered}
$$

and so by adding and integrating with respect to $t$ from 0 to $T$,

$$
E_{1}(T)+\int_{0}^{T}\left(\partial_{t} \partial_{x} \theta^{N}, \partial_{t} \partial_{x} \theta^{N}\right) d t=E_{1}(0)
$$

where

$$
E_{1}(t):=\frac{1}{2}\left\{\left(\partial_{t} \theta^{N}, \partial_{t} \theta^{N}\right)+\varepsilon^{2}\left(\partial_{t}^{2} u^{N}, \partial_{t}^{2} u^{N}\right)+\left(\partial_{t} \partial_{x} u^{N}, \partial_{t} \partial_{x} u^{N}\right)\right\}
$$

5. $\varepsilon$-Asymptotics. We examine the $\varepsilon$-dependence of the solution of the IVP (3.3)(3.5) for fixed $N$. First, we eliminate $\Theta^{N}$ from (3.3)-(3.4) by differentiation to get for $\mathbf{U}=\mathbf{U}^{N}$

$$
\varepsilon^{2} \ddot{\mathbf{U}}+\varepsilon^{2} C G \ddot{\mathbf{U}}+C \dot{\mathbf{U}}+C \Lambda \mathbf{U}=\mathbf{0}
$$

where

$$
C:=V^{-1}(I-\Omega) \Omega^{-1}, \quad G:=\Lambda(I-\Omega)^{-1} V .
$$

Based on our experience in [5] we make the following Ansatz for $\mathbf{U}$ :

$$
\mathbf{U}=e^{-t\left(\Sigma+\frac{i}{\varepsilon} T\right)} \mathbf{a}(\varepsilon)+e^{-t\left(\Sigma-\frac{i}{\varepsilon} T\right)} \mathbf{b}(\varepsilon)+e^{-t P} \mathbf{c}(\varepsilon)
$$


where the real matrices $P, \Sigma, T$ and the vectors $\mathbf{a}, \mathbf{b}, \mathbf{c}$ depend on $\varepsilon$ in a way that we indicate below and with $\mathbf{a}(\varepsilon)+\mathbf{b}(\varepsilon)$ real, $\mathbf{a}(\varepsilon)-\mathbf{b}(\varepsilon)$ pure imaginary.

The solution $e^{-t P} \mathbf{c}(\varepsilon)$ is a purely diffusive term while the first two terms in (5.2) correspond to damped waves. The sum of the first two terms will yield a real vector as is required. The vector $\mathbf{U}$ is a solution of (5.1) if and only if $P$ and $\left(\Sigma \pm \frac{i}{\varepsilon} T\right)$ are solutions of the matrix polynomial equation

$$
-\varepsilon^{2} Z^{3}+\varepsilon^{2} C G Z^{2}-C Z+C \Lambda=\mathbf{0}
$$

for the matrix $Z$ with arbitrary vectors $\mathbf{a}(\varepsilon), \mathbf{b}(\varepsilon)$, and $\mathbf{c}(\varepsilon)$. It is easy to see that if $\boldsymbol{\Theta}$ is defined via (3.4) by

$$
\boldsymbol{\Theta}(t ; \varepsilon)=\mathbf{U}(t ; \varepsilon)+\varepsilon^{2}(I-\Omega)^{-1} V \ddot{\mathbf{U}}(t ; \varepsilon),
$$

then the pair $\{\boldsymbol{\Theta}, \mathbf{U}\}$ are solutions of (3.3)-(3.4) if and only if $\mathbf{U}$ is a solution of (5.1). The $\{\mathbf{a}(\varepsilon), \mathbf{b}(\varepsilon), \mathbf{c}(\varepsilon)\}$ will be chosen so that the initial conditions (3.5) are satisfied.

From preliminary computations we are led to choose the following $\varepsilon$ dependence:

$$
\begin{aligned}
& P=P_{0}+\varepsilon^{2} P_{1}+O\left(\varepsilon^{4}\right), \\
& \Sigma=\Sigma_{0}+\varepsilon^{2} \Sigma_{1}+O\left(\varepsilon^{4}\right), \\
& T=T_{0}+\varepsilon^{2} T_{1}+O\left(\varepsilon^{4}\right)
\end{aligned}
$$

and further,

$$
\begin{aligned}
& \mathbf{a}(\varepsilon)=\mathbf{a}^{0}+i \varepsilon \mathbf{a}^{1}+\varepsilon^{2} \mathbf{a}^{2}+i \varepsilon^{3} \mathbf{a}^{3}+O\left(\varepsilon^{4}\right), \\
& \mathbf{b}(\varepsilon)=\mathbf{b}^{0}+i \varepsilon \mathbf{b}^{1}+\varepsilon^{2} \mathbf{b}^{2}+i \varepsilon^{3} \mathbf{b}^{3}+O\left(\varepsilon^{4}\right), \\
& \mathbf{c}(\varepsilon)=\mathbf{c}^{0}+\varepsilon^{2} \mathbf{c}^{2}+O\left(\varepsilon^{4}\right) .
\end{aligned}
$$

The appropriateness of the above Ansätze will be borne out in what follows.

The computations for the diffusive terms and damped wave terms can proceed independently, and we simplify the procedure by doing so. The diffusive term is simpler to treat and we begin with it.

Substitution of

$$
\mathbf{U}(t)=e^{-t P} \mathbf{c}(\varepsilon)
$$

into (5.1), taking into account our assumptions (5.4)-(5.5), yields upon comparing powers of $\varepsilon$

$$
\begin{gathered}
-C P_{0}+C \Lambda=\mathbf{0}, \\
-C P_{1}+C G P_{0}^{2}-P_{0}^{3}=\mathbf{0} \\
-C P_{2}+C G\left(P_{0} P_{1}+P_{1} P_{0}\right)-\left(P_{1} P_{0}^{2}+P_{0} P_{1} P_{0}+P_{0}^{2} P_{1}\right)=\mathbf{0},
\end{gathered}
$$

and so forth. This process can be continued to arbitrary order to determine $P_{0}, P_{1}, P_{2}, \ldots$ in a unique way. Thus we have

$$
P_{0}=\Lambda, \quad P_{1}=\left(G-C^{-1} \Lambda\right) \Lambda^{2},
$$

and so forth.

In a similar way, substitution of

$$
\mathbf{U}(t)=e^{-t\left(\Sigma+\frac{i}{\varepsilon} T\right)} \mathbf{a}(\varepsilon)
$$


into (5.1) yields

$$
\begin{gathered}
T_{0}^{3}-C T_{0}=\mathbf{0}, \\
\left\{\Sigma_{0} T_{0}^{2}+T_{0} \Sigma_{0} T_{0}+T_{0}^{2} \Sigma_{0}-C \Sigma_{0}\right\}-C G T_{0}^{2}+C \Lambda=\mathbf{0}, \\
\left\{T_{1} T_{0}^{2}+T_{0} T_{1} T_{0}+T_{0}^{2} T_{1}-C T_{1}\right\}+C G\left(T_{0} \Sigma_{0}+\Sigma_{0} T_{0}\right)-\left(T_{0} \Sigma_{0}^{2}+\Sigma_{0} T_{0} \Sigma_{0}+\Sigma_{0}^{2} T_{0}\right)=\mathbf{0}, \\
\left\{\Sigma_{1} T_{0}^{2}+T_{0} \Sigma_{1} T_{0}+T_{0}^{2} \Sigma_{1}-C \Sigma_{1}\right\}-C G\left(T_{0} T_{1}+T_{1} T_{0}-\Sigma_{0}^{2}\right) \\
+\left(T_{1} T_{0} \Sigma_{0}+T_{1} \Sigma_{0} T_{0}+\Sigma_{0} T_{1} T_{0}+\Sigma_{0} T_{0} T_{1}+T_{0} T_{1} \Sigma_{0}+T_{0} \Sigma_{0} T_{1}\right)-\Sigma_{0}^{3}=\mathbf{0},
\end{gathered}
$$

and so forth. The first of these four equations gives $T_{0}$ as the unique "positive" square root of $C$ (the other root $T_{0}=\mathbf{0}$ merely reflects the fact that there is also the purely real solution $P_{0}$ obtained above): $T_{0}=C^{1 / 2}$ is obtained as follows.

First note that we can show that the symmetric matrix $(I-\Omega) \Omega^{-1}=\left(\Omega^{-1}-I\right)$ is positive definite. Indeed, by simultaneous diagonalization via congruence: $(I-\Omega)=$ $S D S^{\mathrm{T}}, \Omega=S S^{\mathrm{T}}$ for some nonsingular $S$ and $D=\operatorname{diag}\left(d_{1}, \ldots, d_{N}\right)$ with $d_{j}>0$ (because $(I-\Omega)$ is positive definite). Hence $W:=(I-\Omega) \Omega^{-1}=S D S^{-1}$ so that the symmetric matrix $W$ has only positive eigenvalues and thus is positive definite. Then by simultaneous diagonalization of $W$ and $V$ via congruence: $W=S^{\mathrm{T}} D S, V=S^{\mathrm{T}} S$ with a different $S$ and $D$ but with exactly the same properties as above. Thus $C=V^{-1} W=S^{-1} D S$, i.e., $C$ is similar to a diagonal matrix with positive entries ( $V$ and $W$ do not commute and so we do not claim that $C$ is symmetric). Finally, we chose $C^{1 / 2}:=S^{-1} \sqrt{D} S$ where $\sqrt{D}$ is the unique positive square root of $D$ (not one of the other $\left(2^{N}-1\right.$ ) choices of square roots of $D$ ).

The structure of the remaining three equations (and indeed of the corresponding equations to any order) is the same, viz. successively the new unknown $\left(\Sigma_{0}\right.$ in the second equation, $T_{1}$ in the third, and $\Sigma_{1}$ in the fourth) appears only in the curly brackets with remaining terms known. Moreover, the last two terms in each of the curly brackets cancel by the above choice of $T_{0}$. Thus from the second equation,

$$
\Sigma_{0} T_{0}^{2}+T_{0} \Sigma_{0} T_{0}=C G T_{0}^{2}-C \Lambda=C \Lambda \Omega^{-1}(I-\Omega)
$$

where the right-hand side is known. Since $T_{0}$ is invertible we have

$$
\Sigma_{0} T_{0}+T_{0} \Sigma_{0}=C G T_{0}-C \Lambda T_{0}^{-1}=C \Lambda V T_{0} .
$$

Similarly the third (resp. fourth) equation lead to

$$
\begin{aligned}
& T_{1} T_{0}+T_{0} T_{1}=\text { known terms, } \\
& \Sigma_{1} T_{0}+T_{0} \Sigma_{1}=\text { known terms. }
\end{aligned}
$$

Hence we wish to solve the matrix equation

$$
X A+A X=B
$$

for unknown $X$ with given $B$ and nonsingular $A=T_{0}$. But such a matrix equation is uniquely solvable (see, e.g., [6, vol. 2 ; p. 225]). 
With $P_{j}, \Sigma_{j}$, and $T_{j}$ now determined for $j=0,1$ (and for $j>1$ if we wish to go to higher order) we turn now to $\mathbf{a}^{j}, \mathbf{b}^{j}$, and $\mathbf{c}^{j}$. Our Ansatz (5.2)-(5.3) applied to the initial conditions (3.5) yields the linear algebraic system

$$
\begin{gathered}
\mathbf{a}(\varepsilon)+\mathbf{b}(\varepsilon)+\mathbf{c}(\varepsilon)=\mathbf{f}^{N} \\
-\left(\Sigma+\frac{i}{\varepsilon} T\right) \mathbf{a}(\varepsilon)-\left(\Sigma-\frac{i}{\varepsilon} T\right) \mathbf{b}(\varepsilon)-P \mathbf{c}(\varepsilon)=\mathbf{g}^{N} \\
\varepsilon^{2}\left\{\left(\Sigma+\frac{i}{\varepsilon} T\right)^{2} \mathbf{a}(\varepsilon)+\left(\Sigma-\frac{i}{\varepsilon} T\right)^{2} \mathbf{b}(\varepsilon)+P^{2} \mathbf{c}(\varepsilon)\right\}=V^{-1}(I-\Omega)\left[\phi^{N}-\mathbf{f}^{N}\right]
\end{gathered}
$$

for the vectors $\mathbf{a}(\varepsilon), \mathbf{b}(\varepsilon), \mathbf{c}(\varepsilon)$. As was done for $P, \Sigma$, and $T$ one can determine successively from (5.6) the $\mathbf{a}^{i}, \mathbf{b}^{i}$, and $\mathbf{c}^{j}$ in (5.5) to any order in $\varepsilon$ as follows.

The second equation in (5.6) yields directly that

$$
T_{0}\left(\mathbf{a}^{0}-\mathbf{b}^{0}\right)=\mathbf{0}
$$

so that $\mathbf{a}^{0}=\mathbf{b}^{0}$. Then the third equation in (5.6) yields

$$
-2 T_{0}^{2} \mathbf{a}^{0}=V^{-1}(I-\Omega)\left[\phi^{N}-\mathbf{f}^{N}\right]
$$

so that

$$
\mathbf{a}^{0}=\mathbf{b}^{0}=\frac{1}{2} \Omega\left(\mathbf{f}^{N}-\phi^{N}\right),
$$

and then the first equation in (5.6) yields

$$
\mathbf{c}^{0}=\Omega \phi^{N}+(I-\Omega) \mathbf{f}^{N} .
$$

The higher-order coefficients are determined successively in a similar way to any order in $\varepsilon$. In particular, one finds

$$
-\mathbf{b}^{1}=\mathbf{a}^{1}=\frac{1}{2} T_{0}^{-1}\left\{\mathbf{g}^{N}+P_{0} \mathbf{c}^{0}+2 \Sigma_{0} \mathbf{a}^{0}\right\}
$$

and

$$
\mathbf{b}^{2}=-\frac{1}{2} \mathbf{c}^{2}=\mathbf{a}^{2}=\frac{1}{2} C^{-1}\left\{P_{0}^{2} \mathbf{c}^{0}+2 \Sigma_{0}^{2} \mathbf{a}^{0}-2\left(T_{0} T_{1}+T_{1} T_{0}\right) \mathbf{a}^{0}-2\left(T_{0} \Sigma_{0}+\Sigma_{0} T_{0}\right) \mathbf{a}^{1}\right\} .
$$

We now have completely determined

$$
\begin{aligned}
\mathbf{U}^{N, 2}(t ; \varepsilon)= & \exp \left\{-t\left[\frac{i}{\varepsilon} T_{0}+\Sigma_{0}+i \varepsilon T_{1}+\varepsilon^{2} \Sigma_{1}\right]\right\}\left[\mathbf{a}^{0}+i \varepsilon \mathbf{a}^{1}+\varepsilon^{2} \mathbf{a}^{2}\right] \\
& +\exp \left\{-t\left[-\frac{i}{\varepsilon} T_{0}+\Sigma_{0}-i \varepsilon T_{1}+\varepsilon^{2} \Sigma_{1}\right]\right\}\left[\mathbf{b}^{0}+i \varepsilon \mathbf{b}^{1}+\varepsilon^{2} \mathbf{b}^{2}\right] \\
& +\exp \left\{-t\left[P_{0}+\varepsilon^{2} P_{2}\right]\right\}\left[\mathbf{c}^{0}+\varepsilon^{2} \mathbf{c}^{2}\right]
\end{aligned}
$$

and

$$
\Theta^{N, 2}(t ; \varepsilon)=\mathbf{U}^{N, 2}(t ; \varepsilon)+\varepsilon^{2}(I-\Omega)^{-1} V \ddot{\mathbf{U}}^{N, 2}
$$

which, to the indicated orders in $\varepsilon$, satisfy (3.3)-(3.5)

$$
\begin{gathered}
\widetilde{\Pi}\left[\boldsymbol{\Theta}^{N .2}, \mathbf{U}^{N, 2}\right]=-\varepsilon^{3} \Omega \rho(t ; \varepsilon), \\
\widetilde{H}_{\varepsilon}\left[\boldsymbol{\Theta}^{N, 2}, \mathbf{U}^{N, 2}\right]=\mathbf{0}
\end{gathered}
$$


uniformly in $t$ for $t$ in $[0, T]$ and

$$
\begin{gathered}
\mathbf{U}^{N, 2}(0 ; \varepsilon)=\mathbf{f}^{N}, \quad \dot{\mathbf{U}}^{N, 2}(0 ; \varepsilon)=\mathbf{g}^{N}, \\
\boldsymbol{\Theta}^{N, 2}(0 ; \varepsilon)=\phi^{N}+O\left(\varepsilon^{4}\right) .
\end{gathered}
$$

Here

$$
\begin{aligned}
\boldsymbol{\rho}(t ; \varepsilon)= & (I-\Omega)^{-1} V\left\{M_{+} \exp \left\{-t\left[\frac{i T_{0}}{\varepsilon}+\Sigma_{0}+i \varepsilon T_{1}+\varepsilon^{2} \Sigma_{1}\right]\right\}\left(\mathbf{a}^{0}+i \varepsilon \mathbf{a}^{1}+\varepsilon^{2} \mathbf{a}^{2}\right)\right. \\
& +M_{-} \exp \left\{-t\left[-\frac{i T_{0}}{\varepsilon}+\Sigma_{0}-i \varepsilon T_{1}+\varepsilon^{2} \Sigma_{1}\right]\right\}\left(\mathbf{b}^{0}+i \varepsilon \mathbf{b}^{1}+\varepsilon^{2} \mathbf{b}^{2}\right) \\
& \left.+\varepsilon M \exp \left\{-t\left[P_{0}+\varepsilon^{2} P_{1}\right]\right\}\left(\mathbf{c}^{0}+\varepsilon^{2} \mathbf{c}^{2}\right)\right\}
\end{aligned}
$$

where $M_{+}, M_{-}(\operatorname{resp} . M)$ are matrix polynomials in $T_{0}, \Sigma_{0}, T_{1}, \Sigma_{1}$ (resp. $\left.P_{0}, P_{1}\right)$ of degree 3 with coefficients that have nonnegative integer powers of $\varepsilon$. Note that Eq. (3.4) is satisfied to all orders in $\varepsilon$ simply because of the definition of $\Theta^{N, 2}$.

Let us note that, in contrast to the C/QS approximation of the temperature and displacement, which contain only diffusive terms, the Galerkin coefficients $\Theta^{N, 2}$ and $\mathbf{U}^{N, 2}$ even to lowest order in $\varepsilon$ contain both diffusive and damped wave terms. If we examine the corresponding Galerkin coefficient $\mathbf{H}^{N}$ for the entropy we find (perhaps surprisingly) that it has only diffusive behavior to lowest order in $\varepsilon$ as we now show.

First define the entropy $\eta^{N}$ in consonance with (2.8):

$$
\eta^{N}(x, t ; \varepsilon):=\theta^{N}(x, t ; \varepsilon)+\gamma u_{x}^{N}(x, t ; \varepsilon) .
$$

Then, because of (2.8), expand $\eta^{N}$ in terms of $\left\{A w_{l}\right\}$ :

$$
\eta^{N}(x, t ; \varepsilon)=\sum_{l=1}^{N} H_{l}^{N}(t ; \varepsilon) A w_{l},
$$

so that taking the inner product with $w_{m}$ yields

$$
\mathbf{H}^{N}=\Omega \Theta^{N}+(I-\Omega) \mathbf{U}^{N}
$$

for the vector $\mathbf{H}^{N}$ with components $H_{l}^{N}$ and correspondingly

$$
\mathbf{H}^{N, 2}=\Omega \Theta^{N, 2}+(I-\Omega) \mathbf{U}^{N, 2}
$$

for our asymptotic (in $\varepsilon$ ) approximation. Using (5.3),

$$
\begin{aligned}
\mathbf{H}^{N, 2} & =\mathbf{U}^{N, 2}+\varepsilon^{2} \Omega(I-\Omega)^{-1} V \ddot{\mathbf{U}}^{N, 2} \\
& =e^{-t\left(P_{0}+\varepsilon^{2} P_{1}\right)}\left[\mathbf{c}^{0}+\varepsilon^{2} \mathbf{c}^{2}\right] \\
& =e^{-t \Lambda} \mathbf{c}^{0}+O(\varepsilon)
\end{aligned}
$$

so that to the lowest order in $\varepsilon$ (viz. $\varepsilon^{0}$ ) the Galerkin approximation of entropy consists of pure diffusion. Moreover, $\eta^{N}$ to the lowest order in $\varepsilon$ corresponds exactly to the C/QS 
entropy with initial value $\hat{\eta}(x, 0)=\phi(x)+\gamma f^{\prime}(x)$. Indeed, using the asymptotic result $(5.7)$,

$$
\begin{aligned}
\eta^{N}(x, t ; \varepsilon) & =\sum_{l=1}^{N} H_{l}^{N} A w_{l} \sim \sum_{l=1}^{N} e^{-\lambda_{l} t} c_{l}^{0} A w_{l} \quad\left(\varepsilon \rightarrow 0^{+}\right) \\
& =\sum_{l=1}^{N} e^{-\lambda_{l} t}\left\{\sum_{m=1}^{N}\left(\phi_{m}^{N} w_{m}+\gamma f_{m}^{N} v_{m}^{\prime}, w_{l}\right)\right\} A w_{l} \\
& =\sum_{l=1}^{N} e^{-\lambda_{l} t}\left(\phi^{N}+\gamma \frac{d}{d x} f^{N}, w_{l}\right) A w_{l}
\end{aligned}
$$

which is precisely (2.9) when truncated at order $N$.

6. Asymptotic correctness. The above formal asymptotics seem to indicate that

$$
\begin{aligned}
& \theta^{N}(x, t ; \varepsilon)=\theta^{N, 2}(x, t ; \varepsilon)+O\left(\varepsilon^{\alpha}\right), \\
& u^{N}(x, t ; \varepsilon)=u^{N, 2}(x, t ; \varepsilon)+O\left(\varepsilon^{\alpha}\right)
\end{aligned}
$$

for some positive $\alpha$, uniformly in $(x, t)$ for $(x, t)$ in $[0,1] \times[0, T]$. In fact, this is rigorously correct as we will show below.

Here, of course

$$
\theta^{N, 2}(x, t ; \varepsilon):=\sum_{l=1}^{N} \Theta_{l}^{N, 2}(t ; \varepsilon) \omega_{l}(x), \quad u^{N, 2}(x, t ; \varepsilon):=\sum_{l=1}^{N} U_{l}^{N, 2}(t ; \varepsilon) v_{l}(x) .
$$

In this regard let us note that it is easy to see that

$$
\exp \left\{-t\left[P_{0}+\varepsilon^{2} P_{1}\right]\right\}=e^{-t P_{0}}\left(I+O\left(\varepsilon^{2}\right)\right)
$$

even though $P_{0}$ and $P_{1}$ do not commute, but the corresponding assertion

$$
\exp \left\{-t\left[\frac{i}{\varepsilon} T_{0}+\Sigma_{0}+i \varepsilon T_{0}+\varepsilon^{2} \Sigma_{1}\right]\right\} \stackrel{?}{=} \exp \left\{-t\left[\frac{i}{\varepsilon} T_{0}+\Sigma_{0}+i \varepsilon T_{1}\right]\right\}(I+O(\varepsilon))
$$

is false if for example $T_{0}$ and $\Sigma_{1}$ do not commute. This fact complicates verifying the asymptotic result (6.1)-(6.2).

To show (6.1)-(6.2) let

$$
\begin{aligned}
& \zeta(x, t ; \varepsilon):=\theta^{N}(x, t ; \varepsilon)-\theta^{N, 2}(x, t ; \varepsilon), \\
& z(x, t ; \varepsilon):=u^{N}(x, t ; \varepsilon)-u^{N, 2}(x, t ; \varepsilon) .
\end{aligned}
$$

Then from the fact that

$$
\begin{gathered}
\widetilde{\Pi}\left[\boldsymbol{\Theta}^{N, 2}, \mathbf{U}^{N, 2}\right]=-\varepsilon^{3} \Omega \boldsymbol{\rho}(t ; \varepsilon), \\
\widetilde{H}_{\varepsilon}\left[\boldsymbol{\Theta}^{N, 2}, \mathbf{U}^{N, 2}\right]=\mathbf{0}
\end{gathered}
$$

it follows by a computation as in Sec. 4 (see (4.1)-(4.2)) that

$$
\begin{gathered}
\left(\zeta_{t}, \omega_{m}\right)+\left(\zeta_{x}, \omega_{m}^{\prime}\right)-\gamma\left(z_{t}, \omega_{m}^{\prime}\right)=\varepsilon^{3} \sum_{l=1}^{N} \rho_{l}\left(\omega_{l}, \omega_{m}\right) \\
\varepsilon^{2}\left(z_{t t}, v_{m}\right)+\left(z_{x}, v_{m}^{\prime}\right)+\gamma\left(\zeta_{x}, v_{m}\right)=0
\end{gathered}
$$


for $m=1, \ldots, N$ and $\rho_{l}=O(1)$ as $\varepsilon \rightarrow 0^{+}$. Hence by a computation analogous to that leading to (4.3), we have with $\rho:=\sum_{l=1}^{N} \rho_{l} \omega_{l}$,

$$
\begin{gathered}
\left(\zeta_{t}, \zeta\right)+\left(\zeta_{x}, \zeta_{x}\right)-\gamma\left(z_{t}, \zeta_{x}\right)=\varepsilon^{3}(\rho, \zeta), \\
\varepsilon^{2}\left(z_{t t}, z_{t}\right)+\left(z_{x}, z_{x t}\right)+\gamma\left(\zeta_{x}, z_{t}\right)=0,
\end{gathered}
$$

and so we have the Energy Identity

$$
E(T)+\int_{0}^{T}\left(\zeta_{x}, \zeta_{x}\right) d t=E(0)+\varepsilon^{3} \int_{0}^{T}(\rho, \zeta) d t
$$

where $\rho=\rho(x, t ; \varepsilon)=O(1)$ uniformly in $(x, t)$ for $(x, t)$ in $[0,1] \times[0, T]$ and

$$
E(t):=\frac{1}{2}\left\{(\zeta, \zeta)+\varepsilon^{2}\left(z_{t}, z_{t}\right)+\left(z_{x}, z_{x}\right)\right\}
$$

We now estimate the integral term in (6.7):

$$
\left|\varepsilon^{3} \int_{0}^{T}(\rho, \zeta) d t\right| \leq \frac{1}{2} \varepsilon^{6} \int_{0}^{T}(\rho, \rho) d t+\frac{1}{2} \int_{0}^{T}(\zeta, \zeta) d t
$$

by application of the Schwarz inequality and the arithmetic-geometric mean inequality. The integrand in the last integral is in turn majorized by $E(t)$. Hence

$$
E(T)+\int_{0}^{T}\left(\zeta_{x}, \zeta_{x}\right) d t \leq\left[E(0)+\frac{\varepsilon^{6}}{2} \int_{0}^{T}(\rho, \rho) d t\right]+\int_{0}^{T} E(t) d t
$$

and so by Gronwall's inequality

$$
E(t) \leq\left[E(0)+\frac{\varepsilon^{6}}{2} \int_{0}^{T}(\rho, \rho) d t\right] e^{T}
$$

We show next that the last integral is bounded (as $\varepsilon$ tends to zero). We have

$$
\left|\int_{0}^{T}(\rho, \rho) d t\right| \leq \int_{0}^{T}\left|\rho^{\mathrm{T}} \Omega \rho\right| d t \leq\|\Omega\| \int_{0}^{T}\|\rho\|^{2} d t
$$

for any matrix norm compatible with the corresponding vector norm. Moreover, it is sufficient to estimate separately each of the three pieces that make up $\rho$ in (5.10). The estimate of the pure diffusion term (the third term in (5.10)) is immediate because of (6.3). For the first (damped wave) term in (5.10), it is sufficient to show that the matrix exponential

$$
\exp \left\{-t\left[i T_{0} / \varepsilon+R\right]\right\}
$$

is bounded as $\varepsilon$ tends to zero for a constant (in $t$ ) matrix $R$ that is $O(1)$ in $\varepsilon$. Now recalling from Sec. 5 that $T_{0}=S^{-1} \sqrt{D} S$ it follows that $\exp \left\{-i t T_{0} / \varepsilon\right\}$ is $O(1)$ for all $t$. Further, the matrix exponential in (6.10) is the solution $X$ of the IVP consisting of the matrix differential equation

$$
\dot{X}+\frac{i}{\varepsilon} T_{0} X=-R X
$$

with initial condition $X(0)=I$. Hence

$$
X(t)=e^{-i t T_{0} / \varepsilon}-\int_{0}^{t} e^{-i(t-s) T_{0} / \varepsilon} R X(s) d s
$$


and thus

$$
\|X(t)\| \leq k_{1}+k_{1}\|R\| \int_{0}^{t}\|X(s)\| d s
$$

so that by Gronwall's inequality

$$
\left\|\exp \left\{-t\left[\frac{i T_{0}}{\varepsilon}+R\right]\right\}\right\|=\|X(t)\| \leq k_{1} e^{k T} .
$$

The same estimate is valid for the second (damped wave) time in (5.10). Accordingly an estimate of $E(0)$ by use of the initial conditions (3.5) and (5.9) yields the result that the RHS of $(6.9)$ is $O\left(\varepsilon^{6}\right)$. It follows that $\zeta, z_{x}$ and $\varepsilon z_{t}$ are $O\left(\varepsilon^{3}\right)$ in the $L_{\infty}(0, T ; \mathcal{H})$ norm. Here $\mathcal{H}$ denotes the Hilbert space $L_{2}(0,1)$. Then

$$
z(x, t ; \varepsilon)=\int_{0}^{x} z_{x}(\xi, t ; \varepsilon) d \xi
$$

results in the elementary Sobolev inequality

$$
|z(x, t ; \varepsilon)|^{2} \leq\left(z_{x}(\cdot, t ; \varepsilon), z_{x}(\cdot, t ; \varepsilon)\right)
$$

and so (6.2) holds with $\alpha=3$. A similar estimate on $\zeta$ in the $L_{2}(0, T ; \mathcal{H})$ norm follows in the same way but for the uniform estimate we seek we proceed differently by a higherorder energy estimate as introduced in Sec. 4. The idea is the same as in [11] for multidimensional estimates.

The first step is very close to that leading up to (4.3), the key difference consisting in the loss of a power of $\varepsilon$ in the differentiation of (5.8), viz.

$$
\Omega \ddot{\Theta}^{N, 2}+\Lambda \dot{\boldsymbol{\Theta}}^{N, 2}+(I-\Omega) \ddot{\mathbf{U}}^{N, 2}=O\left(\varepsilon^{2}\right) .
$$

The result is

$$
E^{1}(t)=O\left(\varepsilon^{4}\right)
$$

where

$$
E^{1}(t):=\frac{1}{2}\left\{\left(\zeta_{t}, \zeta_{t}\right)+\varepsilon^{2}\left(z_{t t}, z_{t t}\right)+\left(z_{x t}, z_{x t}\right)\right\} .
$$

Then from the sum of (6.5) and (6.6),

$$
\left(\zeta_{x}, \zeta_{x}\right)=\varepsilon^{3}(\rho, \zeta)-\varepsilon^{2}\left(z_{t t}, z_{t}\right)-\left(z_{x t}, z_{x t}\right)-\left(\zeta_{t}, \zeta\right)
$$

By our previous estimates, including those just concluded, each of the four terms on the RHS are $O\left(\varepsilon^{4}\right)$ (by the Schwarz and arithmetic-geometric mean inequalities). Thus $\zeta_{x}$ is $O\left(\varepsilon^{2}\right)$ in the $L_{\infty}(0, T ; \mathcal{H})$ norm (whereas previously we had an $O\left(\varepsilon^{3}\right)$ estimate for $\zeta_{x}$ in the $L_{2}(0, T ; \mathcal{H})$ norm $)$.

Now from

$$
\zeta(x, t ; \varepsilon)=\zeta(0, t ; \varepsilon)+\int_{0}^{x} \zeta_{x}(\xi, t ; \varepsilon) d \xi
$$

follows an $O\left(\varepsilon^{2}\right)$ estimate for $\zeta$ in the $L_{\infty}(0, t ; \mathcal{H})$ norm.

Thus from the corresponding elementary Sobolev inequality the result (6.1) holds with $\alpha=2$, uniformly in $(x, t)$ for $(x, t)$ in $[0,1] \times[0, T]$. It should be noted that by using $E^{1}$ in addition to $E$ we require slightly more stringent conditions on our data $\phi, f$, and 
$g$. It is also pointed out that it would be more pleasant to have $\alpha=3$ since $\theta^{N, 2}$ itself contains terms of order $\varepsilon^{2}$.

Finally, let us note that it is fairly obvious from what we have done in this section that we could increase $\alpha$ in (6.1)-(6.2) by replacing $\left\{\theta^{N, 2}, u^{N, 2}\right\}$ by $\left\{\theta^{N, l}, u^{N, l}\right\}$ which denotes the corresponding term using additional $P_{j}, \Sigma_{j}, T_{j}, \mathbf{a}^{j}, \mathbf{b}^{j}$, and $\mathbf{c}^{j}$. This would, of course, necessitate further restrictions on the data.

Appendix A. Convergence of Galerkin approximations. The Galerkin scheme of Sec. 3 is closely tied-in with a weak formulation of the IBVP (1.1)-(1.7). For this formulation we have to introduce some preliminaries which are well known for purely parabolic and hyperbolic problems. These approaches are now available in many places (see, e.g., [12]).

By $\mathcal{H}($ resp. $\mathcal{V})$ we denote the Hilbert spaces $L_{2}(0,1)$ (resp. $\left.H_{0}^{1}(0,1)\right)$. The inner product in $\mathcal{V}$ will be denoted $(\cdot, \cdot)_{1}$ while that in $\mathcal{H}$ by our general agreement in Sec. 2 will be denoted $(\cdot, \cdot)$ without subscript. In Sec. 2 we also agreed to indicate the duality pairing in $\mathcal{V}^{*} \times \mathcal{V}$ by $\langle\cdot, \cdot\rangle$.

The weak solution of the IBVP (1.1)-(1.7) is arrived at in the usual way: Multiply a classical solution of (1.1)-(1.2) by suitable test functions and integrate by parts. Here for our time-dependent problem it is convenient to view a solution $\{\theta, u\}$ of $(1.1)-(1.7)$ as a map from $[0, T]$ into $\mathcal{V}$ and we allow the context to make clear whether we are viewing $\theta$ as $\theta=\theta(t)$ (with $\varepsilon$ suppressed for notational simplicity) or $\theta=\theta(x, t ; \varepsilon)$ as we have written above (similarly for $u$ ).

Thus we are led to a weak solution of (1.1)-(1.7) defined as a pair $\{\theta, u\}$ such that

(1) $\theta \in L_{2}(0, T ; \mathcal{V}), \dot{\theta} \in L_{2}\left(0, T ; \mathcal{V}^{*}\right)$;

(2) $u \in L_{2}(0, T ; \mathcal{V}), \dot{u} \in L_{2}(0, T ; \mathcal{H}), \ddot{u} \in L_{2}\left(0, T ; \mathcal{V}^{*}\right)$;

(3) the following traces on $t=0$ exist:

$$
\operatorname{tr} \theta=\phi, \quad \operatorname{tr} u=f, \quad \operatorname{tr} \dot{u}=g ;
$$

(4) for each $\Phi, W$ in $\mathcal{V}$ and each $\zeta, z$ in $C_{0}^{\infty}(0, T ; \mathbb{R})$

$$
\begin{gathered}
\int_{0}^{T}\left\{-(\theta(t), \Phi) \dot{\zeta}(t)+\left(\partial_{x} \theta(t), \partial_{x} \Phi\right) \zeta(t)-\gamma\left(\dot{u}(t), \partial_{x} \Phi\right) \zeta(t)\right\} d t=0 \\
\int_{0}^{T}\left\{\varepsilon^{2}(u(t), W) \ddot{z}(t)+\left(\partial_{x} u(t), \partial_{x} W\right) z(t)+\gamma\left(\partial_{x} \theta(t), W\right) z(t)\right\} d t=0
\end{gathered}
$$

which says that the $t$-derivatives are taken in the sense of distributions.

Our goal is to show the convergence of the Galerkin approximations of Sec. 3, which we write here as

$$
\begin{aligned}
\theta^{N}(t) & :=\sum_{l=1}^{N} \Theta_{l}^{N}(t ; \varepsilon) \omega_{l}, \\
u^{N}(t) & :=\sum_{l=1}^{N} U_{l}^{N}(t ; \varepsilon) v_{l}
\end{aligned}
$$

to a $\{\theta, u\}$ satisfying (i)-(iv) but with slightly nicer regularity properties. Thus we view $\left\{\theta^{N}, u^{N}\right\}$ here as maps from $[0, T]$ into $\mathcal{V}$ (and even nicer spaces when appropriate). For 
later use we write (3.1)-(3.2) here as

$$
\begin{aligned}
\left(\dot{\theta}^{N}(t), \omega_{m}\right)+\left(\partial_{x} \theta^{N}(t), \partial_{x} \omega_{m}\right)-\gamma\left(\dot{u}^{N}(t), \partial_{x} \omega_{m}\right) & =0, \\
\varepsilon^{2}\left(\ddot{u}^{N}(t), v_{m}\right)+\left(\partial_{x} u^{N}(t), \partial_{x} v_{m}\right)+\gamma\left(\partial_{x} \theta^{N}(t), v_{m}\right) & =0 .
\end{aligned}
$$

As always the key to convergence involves appropriate estimates which, for us, are supplied in Sec. 4. In fact, from (4.3) we obtain the boundedness of

$$
\begin{gathered}
\theta^{N}, \varepsilon \dot{u}^{N}, \partial_{x} u^{N} \quad \text { in the } L_{\infty}(0, T ; \mathcal{H}) \text { norm, } \\
\partial_{x} \theta^{N} \quad \text { in the } L_{2}(0, T ; \mathcal{H}) \text { norm, }
\end{gathered}
$$

and from (4.4) the boundedness of

$$
\begin{gathered}
\dot{\theta}^{N}, \varepsilon \ddot{u}^{N}, \partial_{x} \dot{u}^{N} \quad \text { in the } L_{\infty}(0, T ; \mathcal{H}) \text { norm, } \\
\partial_{x} \dot{\theta}^{N} \quad \text { in the } L_{2}(0, T ; \mathcal{H}) \text { norm. }
\end{gathered}
$$

Indeed (A.3)-(A.6) follow from the boundedness, independent of $N$, of $E(0)$ and $E_{1}(0)$; in fact, $E(0)$ and $E_{1}(0)$ are bounded independently of $N$ and $\varepsilon$ for $\varepsilon$ in, say, $(0,1]$. For $E(0)$ this follows from

$$
\left(\theta^{N}(0 ; \varepsilon), \theta^{N}(0 ; \varepsilon)\right)=\left(\Pi^{N} \phi, \Pi^{N} \phi\right) \leq(\phi, \phi)
$$

and the corresponding results for $\varepsilon \dot{u}^{N}$ and $\partial_{x} u^{N}$ provided that $\phi$ and $g$ are in $\mathcal{H}$ and $f$ is in $\mathcal{V}$. For $E^{1}(0)$ we use (3.3) and (3.4) as follows.

From (3.3) we see that $\dot{\theta}^{N}(0 ; \varepsilon)$ is bounded in $L_{2}(0,1)$ independently of $N$ and $\varepsilon$ since $\dot{\boldsymbol{\Theta}}^{N}(0 ; \varepsilon)$ is bounded in terms of $\phi^{N}$ and $\mathbf{g}^{N}$. From $(3.4)$ we have that $\varepsilon^{2} \ddot{\mathbf{U}}^{N}(0 ; \varepsilon)$ is bounded in terms of $\phi^{N}$ and $f^{N}$ but now with greater regularity conditions on $\phi$ and $f$ and thus $\varepsilon \ddot{u}^{N}(0 ; \varepsilon)$ is bounded in the $L_{2}(0,1)$ norm independently of $N$ and $\varepsilon$. Finally, the same is true for $\partial_{x} \dot{u}^{N}(0 ; \varepsilon)$ if $g^{\prime}$ is in $\mathcal{V}$. Hence $E_{1}(0)$ is bounded and our assertion is proved.

Let us note that $u^{N}$, omitted from our list in (A.3)-(A.6), is bounded in the $L_{\infty}(0, T ; \mathcal{H})$ norm (even more including in the $L_{\infty}(0, T ; \mathcal{V})$ norm) because of the elementary Sobolev inequality

$$
\left|u^{N}(x, t ; \varepsilon)\right| \leq\left(\partial_{x} u^{N}, \partial_{x} u^{N}\right)
$$

which follows by integration of $\partial_{x} u^{N}$ and the Schwarz inequality. Note our dual use of the symbol $u^{N}$ as forewarned above.

As a result of our estimates we have that the following sequences are bounded (independently of $N)$ in the $L_{2}(0, T ; \mathcal{H})$ norm:

$$
\left\{\theta^{N}\right\},\left\{\partial_{x} \theta^{N}\right\},\left\{\dot{\theta}^{N}\right\},\left\{\partial_{x} \dot{\theta}^{N}\right\},\left\{u^{N}\right\},\left\{\partial_{x} u^{N}\right\},\left\{\dot{u}^{N}\right\},\left\{\partial_{x} \dot{u}^{N}\right\},\left\{\ddot{u}^{N}\right\}
$$

and thus have weakly convergent subsequences (denoted by the same symbols $\left\{\theta^{N}\right\}$, $\left\{\partial_{x} \theta^{N}\right\}$, etc.) which converge to some elements of $L_{2}(0, T ; \mathcal{H})$ which we denote respectively by $\psi, \partial_{x} \psi, \dot{\psi}, \partial_{x} \dot{\psi}, w, \partial_{x} w, \dot{w}, \partial_{x} \dot{w}$, and $\ddot{w}$ (for a proof that the limit of $\dot{\theta}^{N}$ is indeed $\dot{\psi}$ etc. see, e.g., [12]). Thus the conditions (i)-(ii) above for a weak solution of (1.1)(1.7) are more than met. The condition (iii) for the traces is satisfied since the above convergence properties ensure the existence of the traces of $\theta, u$, and $\dot{u}$ in $t=0$ (e.g., convergence of $\left\{\partial_{x} \dot{u}^{N}\right\}$ and $\left\{\ddot{u}^{N}\right\}$ in $L_{2}(0, T ; \mathcal{H})$ is sufficient for the existence of the trace 
of $\dot{w})$ and, moreover, the traces are the limits of (subsequences of) $\theta^{N}(0), u^{N}(0)$, and $\dot{u}^{N}(0)$ viz. the limits are $\phi, f$, and $g$.

Finally, using (A.1)-(A.2) and letting $N \rightarrow \infty$,

$$
\begin{aligned}
& \int_{0}^{T}\left\{\left(\dot{\psi}(t), \omega_{m}\right)+\left(\partial_{x} \psi(t), \partial_{x} \omega_{m}\right)-\gamma\left(\dot{w}(t), \partial_{x} \omega_{m}\right)\right\} \zeta(t) d t=0 \\
& \int_{0}^{T}\left\{\varepsilon^{2}\left(\ddot{w}(t), v_{m}\right)+\left(\partial_{x} w(t), \partial_{x} v_{m}\right)+\gamma\left(\partial \psi(t), v_{m}\right)\right\} z(t) d t=0
\end{aligned}
$$

for any $\zeta, z$ in $C_{0}^{\infty}(0, T)$ and any fixed $m=1,2, \ldots$ Since $\left\{\omega_{m}\right\},\left\{v_{m}\right\}$ are complete in $\mathcal{V}$ we can replace $\omega_{m}, v_{m}$ respectively by arbitrary $\Phi, W$ in $\mathcal{V}$. Thus, by integration by parts with respect to $t$, we see that condition (iv) is met by $\{\psi, w\}$, which are slightly nicer than the $\{\theta, u\}$ in the definition. Therefore, we have shown existence of a weak solution of (1.1)-(1.7).

Our next task is to show that, under our additional restrictions, there is at most one such $\{\psi, w\}$.

Suppose then that there exists $\{\theta, u\}$ satisfying (i)-(iv) in the definition of weak solution but with $\phi=f=g=0$ and in addition (somewhat redundantly) all of the following belong to the space $L_{2}(0, T ; \mathcal{H}): \theta, \dot{\theta}, \partial_{x} \theta, \partial_{x} \dot{\theta}$ and $u, \dot{u}, \partial_{x} u, \partial_{v} \dot{u}, \ddot{u}$. Then from (iv) and the integration by parts,

$$
\begin{aligned}
-\int_{0}^{T}(\theta(t), \Phi) \dot{\zeta}(t) d t & =\int_{0}^{T}(\dot{\theta}(t), \Phi) \zeta(t) d t, \\
\int_{0}^{T}(u(t), W) \ddot{z}(t) d t & =\int_{0}^{T}(\ddot{u}(t), W) z(t) d t,
\end{aligned}
$$

we have for almost all $t$ in $(0, T)$,

$$
\begin{aligned}
(\dot{\theta}(t), \Phi)+\left(\partial_{x} \theta(t), \partial_{x} \Phi\right)-\gamma\left(\dot{u}(t), \partial_{x} \Phi\right) & =0 \\
\varepsilon^{2}(\ddot{u}(t), W)+\left(\partial_{x} u(t), \partial_{x} W\right)+\gamma\left(\partial_{x} \theta(t), W\right) & =0
\end{aligned}
$$

for all $\Phi, W$ in $\mathcal{V}$. Hence with $\Phi=\theta(t)$ and $W=\dot{u}(t)$, by integration and summing,

$$
0=\int_{0}^{T}\left\{(\dot{\theta}(t), \theta(t))+\varepsilon^{2}(\ddot{u}(t), \dot{u}(t))+\left(\partial_{x} u(t), \partial_{x} \dot{u}(t)\right)+\left(\partial_{x} \theta(t), \partial_{x} \theta(t)\right)\right\} d t
$$

and so, as in Sec. 4,

$$
E(T)+\int_{0}^{T}\left(\partial_{x} \theta(t), \partial_{x} \theta(t)\right) d t=E(0)
$$

where

$$
E(t):=\frac{1}{2}\left\{(\theta(t), \theta(t))+\varepsilon^{2}(\dot{u}(t), \dot{u}(t))+\left(\partial_{x} u(t), \partial_{x} u(t)\right)\right\} .
$$

Now for $t=0$ the first two terms in $E(t)$ are zero from (iii). But the third is also since, under our extra conditions, the trace of $\partial_{x} u(t)$ exists and is zero for $t=0$. Thus $E(0)=0$ and hence $E(t)=0$ for almost all $t$ in $[0, T]$. From this uniqueness follows.

Collecting our previous results we now know that all subsequences of $\left\{\theta^{N}, u^{N}\right\}$ converge to the solution of (1.1)-(1.7). 
Acknowledgment. The author would like to thank B. F. Esham, Jr. for many helpful conversations. I am grateful to the referee for suggestions about points of emphasis in the Introduction.

\section{REFERENCES}

[1] B. A. Boley and J. W. Weiner, Theory of Thermal Stresses, John Wiley and Sons, New York, 1960

[2] C. M. Dafermos, On the existence and the asymptotic stability of solutions to the equations of linear thermoelasticity, Arch. Rat. Mech. Anal. 29, 241-271 (1968)

[3] W. A. Day, Heat conduction within linear thermoelasticity, in Springer Tracts in Natural Philosophy, 30, Springer-Verlag, Berlin, 1985

[4] B. F. Esham, Jr. and R. J. Weinacht, Hyperbolic-parabolic singular perturbations for quasi-linear equations, SIAM J. Math. Anal. 20, 1344-1365 (1989)

[5] B. F. Esham, Jr. and R. J. Weinacht, Singular perturbations and the coupled/quasi-static approximation in linear thermoelasticity, SIAM J. Math. Anal. 25, 1521-1536 (1994)

[6] F. R. Gantmacher, Theory of Matrices, 2 vols., Chelsea, New York, 1959

[7] G. C. Hsiao and R. J. Weinacht, A singularly perturbed Cauchy problem, J. Math. Anal. Appl. 71, 242-250 (1979)

[8] R. Racke and Y. Shibata, Global smooth solutions and asymptotic stability in one-dimensional nonlinear thermoelasticity, Arch. Rat. Mech. Anal. 116, 1-34 (1991)

[9] J. E. Rivera, Decomposition of the displacement vector field and decay rates in linear thermoelasticity, SIAM J. Math. Anal. 24, 390-406 (1991)

[10] M. Slemrod, Global existence, uniqueness and asymptotic stability of classical smooth solutions in one-dimensional non-linear thermoelasticity, Arch. Rat. Mech. Anal. 75, 97-133 (1981)

[11] R. J. Weinacht and B. F. Esham, Jr., Energy Estimates in Thermoelasticity, in Non-linear Problems in Applied Mathematics (in honor of Ivar Stakgold on his 70th Birthday), edited by T. S. Angell, L. Pamela Cook, R. E. Kleinman, and W. E. Olmstead, SIAM, Philadelphia, 1996, pp. 259-268

[12] E. Zeidler, Nonlinear Functional Analysis and its Applications, Vol. II/A, Springer-Verlag, New York, 1990 